\title{
NUMERICAL ANALYSIS OF LIFT GENERATION IN A RADIAL SEGMENTED GAS SEAL
}

\author{
Mihai ARGHIR \\ Institut PPRIME, UPR CNRS 3346 \\ Université de Poitiers, ISAE ENSMA, \\ France
}

\author{
Samia DAHITE \\ Safran Aircraft Engines \\ Villaroche, France
}

\begin{abstract}
A radial segmented seal is composed of three or six carbon segments that are assembled by a circumferential (garter) spring that presses them against the rotor. Assembled, they take the form of an annular ring. Each segment has several pads that generate a radial lift force depending on the rotor speed. There are many ways of creating effective lift forces. For example, a pocket on the pad creates a lift force because each pad will act as a Rayleigh step bearing. A groove on the rotating shaft will also create a radial lift force on the pad. However, this latter lift force will be unsteady. The aim of the present work is the numerical study of the lift created by a grooved rotor on a pad. Due to the very small operating radial clearances of radial segmented seals (less than $10 \mu \mathrm{m}$ ), the problem can be simplified by analyzing a single pad and a grooved runner. Previous analysis of gas face seals or thrust bearings always considered grooved pads and a smooth runner, even when the runner was grooved. The peculiarity of this study, which is the first of its kind, is considering the unsteady problem of the moving runner grooves. The analysis was performed for a single pad of a radial segmented seal operating with air.
\end{abstract}

\section{INTRODUCTION}

Carbon ring seals are widely used in small and medium size rotating machinery due to their low leakage rates and reduced space requirements. There are mainly three categories of carbon ring seals: the face seal, the floating ring seal and the radial segmented seal [1], [2]. They all have a main seal and a secondary seal. The floating ring and the radial segmented seals are closely related because the main leakage flow occurs axially between the rotor surface and the inner surface of the carbon ring (Figure 1). The secondary seal consists of a small size "nose" of the carbon ring continuously in contact with the stator. It is therefore supposed to be completely closed. The radial clearance in the main sealing path of the floating ring seal is of the order of $10 \mu \mathrm{m}$ to $20 \mu \mathrm{m}$.

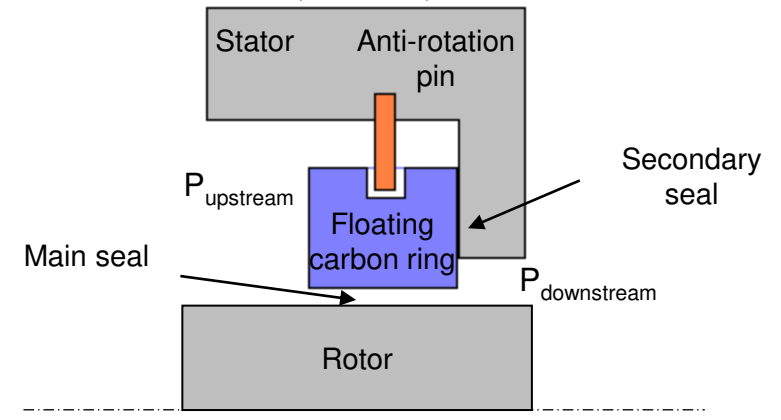

Figure 1 Schematic view of a floating ring seal

This can be too large in some applications. In order to obtain a seal with adjustable clearance, the carbon ring is split in three or six overlapping segments as show in Figure 2. A garter spring pushes the segments against the rotor, therefore when rotation speed is zero the seal is closed. During operation, the seal surface should not be in contact with the rotor in order to prevent precocious wear. Therefore, the segments open and create a very small radial clearance of the order 1 to $5 \mu \mathrm{m}$ or less. The seal opening is possible if a lift force is generated by the rotation speed. Figure 3 shows that the segments are provided with pads separated by deep grooves. The grooves separate individual pads and the lip. The lip (Figure 4) is the main seal but its axial width is very small so only the pads are responsible for creating lift. There are several solutions for creating the lift force under the pads.

-The pads are smooth cylindrical surface (Figure 3a). In this case, a reduced lift effect is created by the waviness errors always present. The radial clearance of the segment under operating conditions will be very small and therefore the seal will wear rapidly. 
- The pads are provided with pockets (Figure 2 and Figure 3b). In this case the lift force is much higher and the seal can operate with a radial clearance large enough to avoid wear.

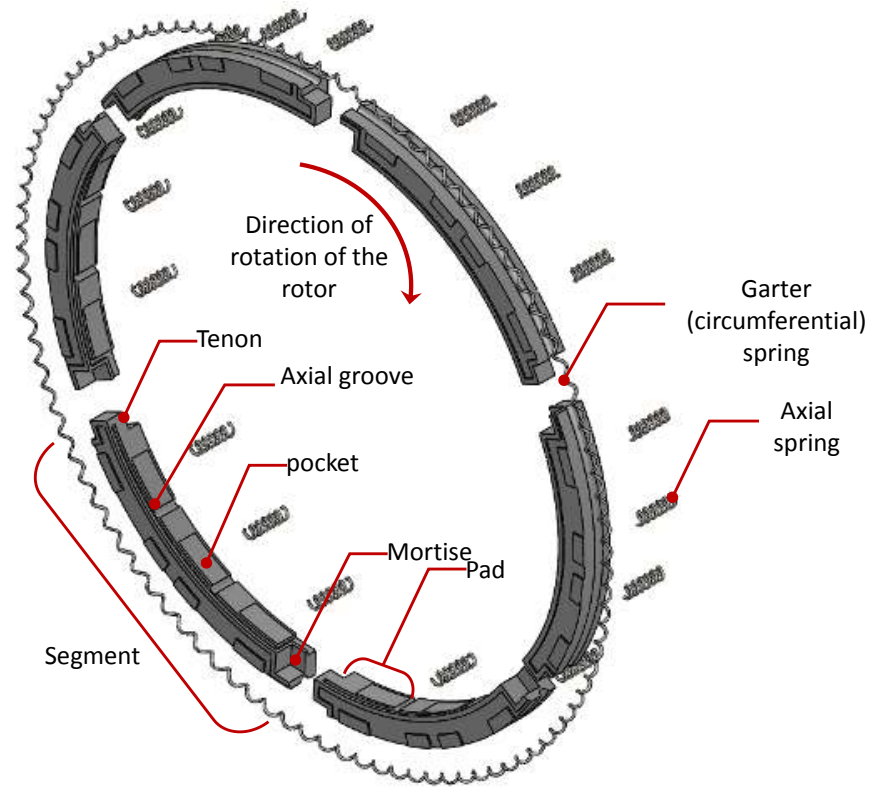

Figure 2 The radial segmented annular seal

- The pads are smooth cylindrical surfaces but the rotor is provided with grooves (Figure 3c). The generated lift force is different from the previous cases because it is unsteady.

The first two cases were analyzed in [3]. The influence of the pockets depth and size on the seal opening and leakage flow was analyzed in depth.

The present work deals with the third case, when a grooved rotor creates the unsteady lift. Two cases were considered. First, when the rotor groove is normal to the segment and second, when the rotor groove is inclined. Thrust bearings with inclined grooves have been studied since long [4], but they were operating against a smooth rotor. Studies involving a grooved runner have also been conducted [5] but they do not consider the effect of moving grooves. Therefore, the present study explores the unsteady lift effect of a grooved rotor. Together with the force applied by the garter spring and the nose friction force, the lift force controls the opening of the seal. It has therefore a first order impact on the leakage flow of the radial segmented seal.

The paper presents the numerical model for calculating the unsteady lift force created by a pad and a rotating groove. The model is detailed for a transversal and for an inclined groove. The results show the lift and the shear forces generated by the pad for different compressibility numbers. For very large compressibility numbers the lift force generated by the inclined groove is $25 \%$ higher.

\section{NOMENCLATURE}

$\begin{array}{ll}h & \text { Height of the fluid film [m] } \\ l_{i j} & \text { Distance between i and } \mathrm{j}[\mathrm{m}]\end{array}$

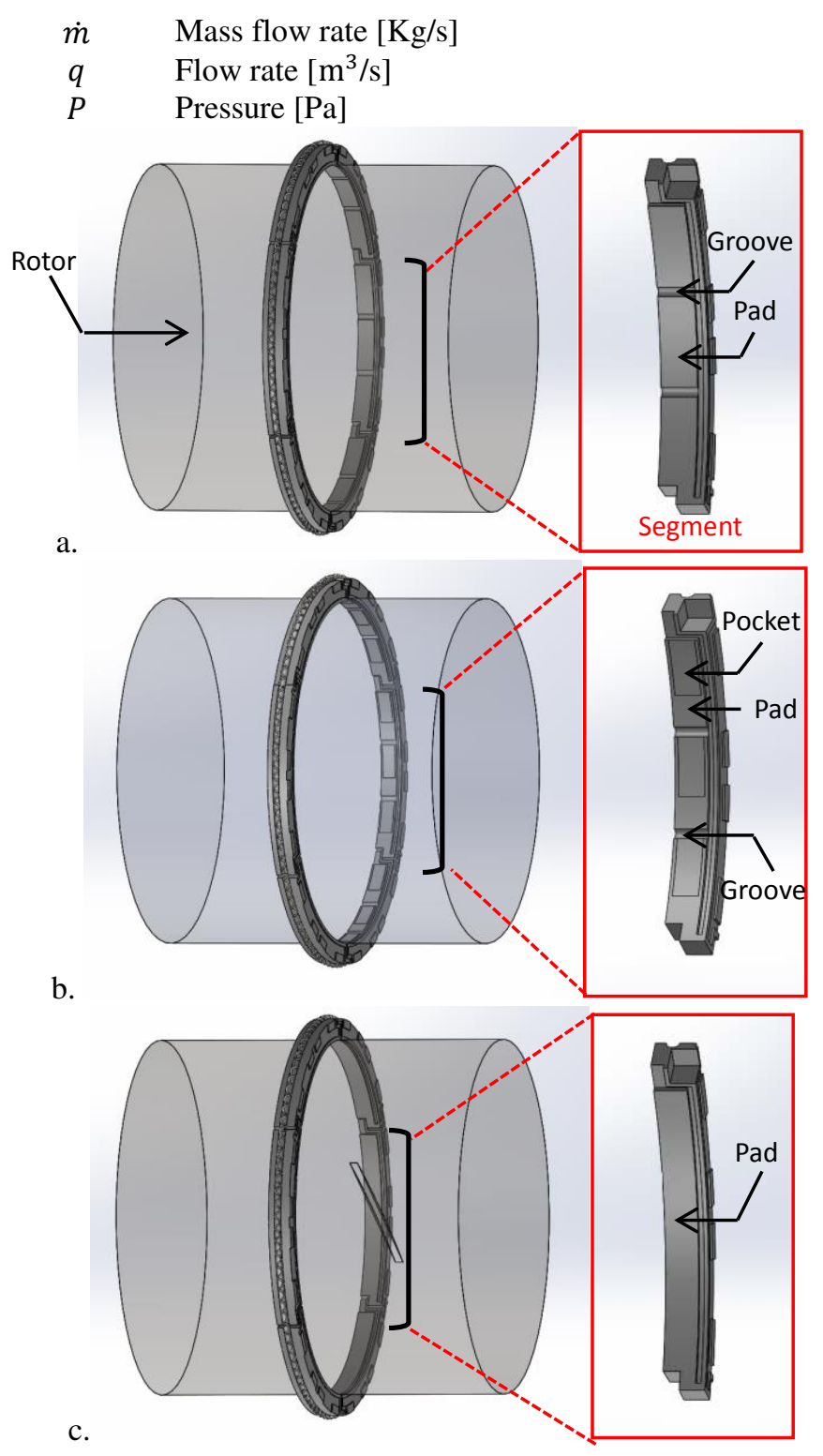

Figure 3 Lift generation in a radial segmented annular seal:

a. segments with transversal grooves and without pockets

b. segments with transversal grooves and pockets

c. smooth segments and grooved rotor

$\tilde{P} \quad$ Upwinded pressure $[\mathrm{Pa}]$

$P_{\text {atm }} \quad$ Atmospheric pressure [Pa]

$t \quad$ Temporal coordinate [s]

$\Delta t \quad$ Time step [s]

$L_{x} \quad$ Pad length [m]

$L_{z} \quad$ Pad width [m]

$U_{x} \quad$ Velocity of the moving surface $[\mathrm{m} / \mathrm{s}]$

$u_{i} \quad$ Fluid velocity on the cell boundary i $[\mathrm{m} / \mathrm{s}]$

$x \quad$ Cartesian coordinate of the axis $\overrightarrow{\mathrm{x}}$

$z \quad$ Cartesian coordinate of the axis $\overrightarrow{\mathrm{z}}$ 

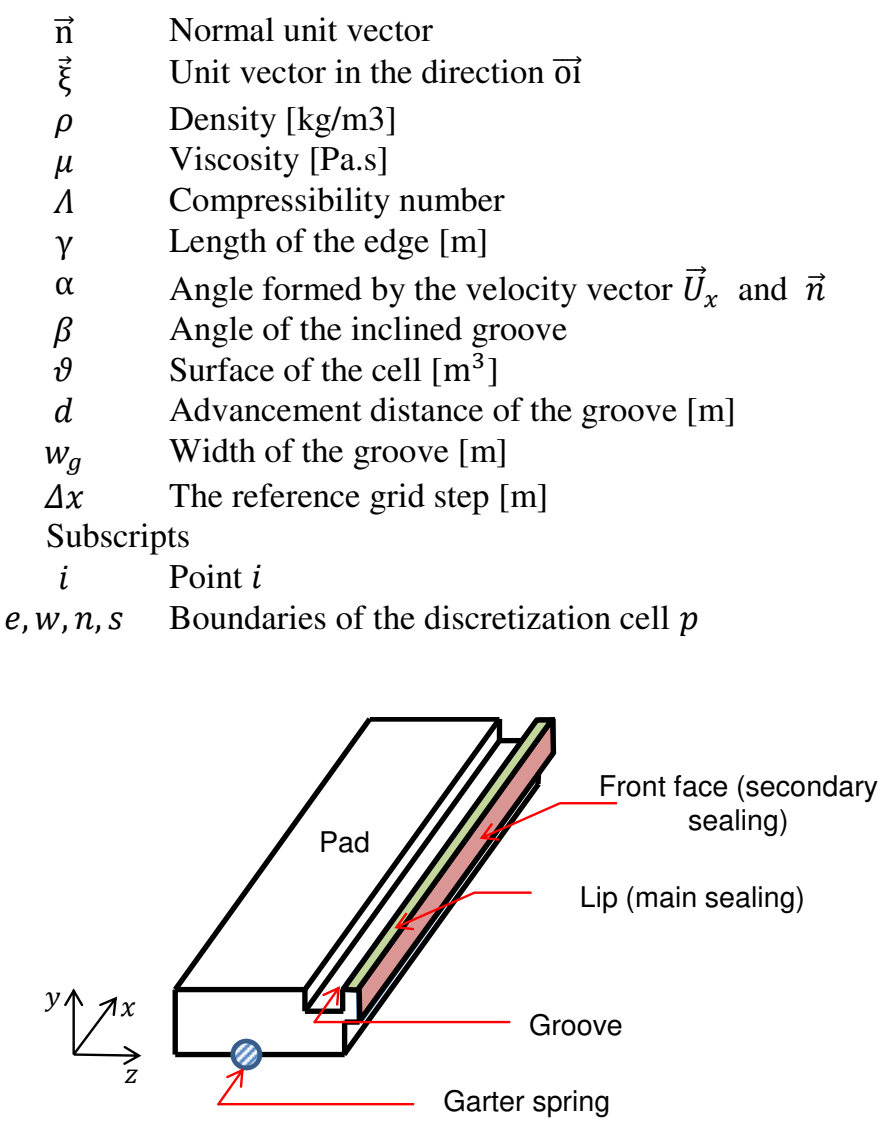

Figure 4 Geometry of the segment

\section{NUMERICAL SOLUTION OF THE REYNOLDS EQUATION}

The analysis was performed for a single pad of a radial segmented seal operating with air treated as a perfect gas. The unsteady, generalized Reynolds equation in Cartesian coordinates $(\vec{x}, \vec{z})$ is:

$\frac{\partial}{\partial x}\left(\frac{\rho h^{3}}{12 \mu} \frac{\partial P}{\partial x}\right)+\frac{\partial}{\partial z}\left(\frac{\rho h^{3}}{12 \mu} \frac{\partial P}{\partial z}\right)=\frac{U_{x}}{2} \frac{\partial(\rho h)}{\partial x}+\frac{\partial(\rho h)}{\partial t}$

It is the supposed that the flow is isothermal and the air obeys the universal state law. These are usual assumptions for thin film flows sufficient for enlightening the qualitative nature of the lifting effects. The densities in eq. (1) are then replaced by pressures.

$\frac{\partial}{\partial x}\left(\frac{\tilde{P} h^{3}}{12 \mu} \frac{\partial P}{\partial x}\right)+\frac{\partial}{\partial z}\left(\frac{\tilde{P} h^{3}}{12 \mu} \frac{\partial P}{\partial z}\right)=\frac{U_{x}}{2} \frac{\partial(\tilde{P} h)}{\partial x}+\frac{\partial(P h)}{\partial t}$

This non-linear partial differential equation must be solved numerically. The numerical solution was adapted to the two investigated cases: when the groove is transversal to the pad and when it is inclined. Although the pad is rectangular, the two cases require different meshes. The case of a transversal groove can be dealt with a rectangular mesh (Figure 5a), but an unstructured mesh must be used when the groove is inclined (Figure 5b).

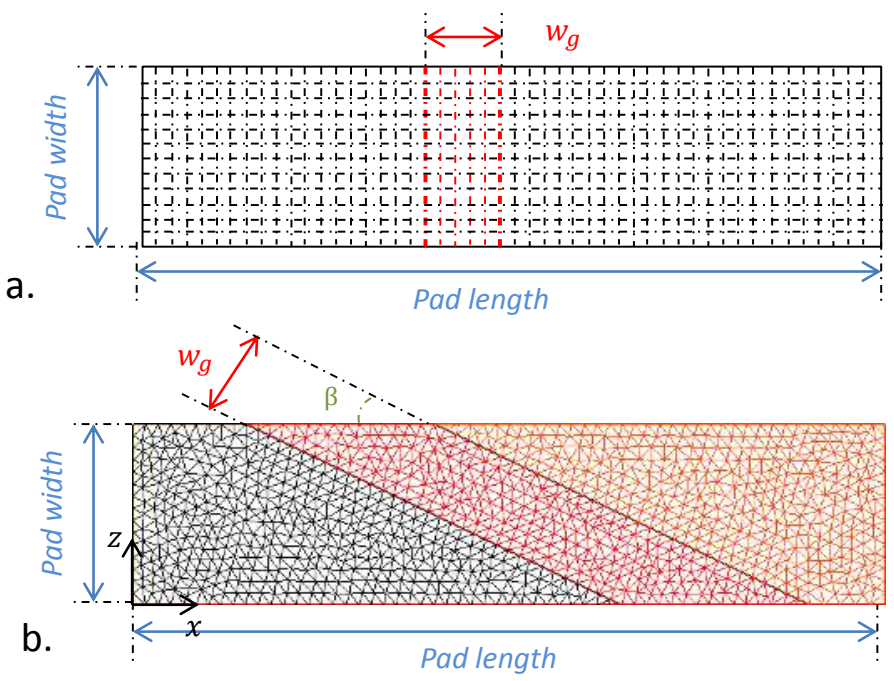

Figure 5 Computational grid and geometry of the pad for a- a transversal groove $b$ - an inclined groove

In both cases, the Reynolds equation was discretized by using a cell centered finite volume method. The numerical method for taking into account discontinuities was developed in [6] and [7] for journal bearings and is now applied for seals.

\section{The structured rectangular grid}

Figure 6 depicts a cell of the rectangular structured mesh used when the rotor grooves are perpendicular to the pads. The subscript $P$ refers to the center of the main cell and the subscripts $E, W, S, N$ designate the east, west, south and north adjacent cells. The lowercase subscripts $e, w, s$ and $n$ refer to cell boundaries. Following the finite volume method ([6], [7]) eq. (2) is integrated over this volume.

$$
\begin{gathered}
\int_{x_{w}}^{x_{e}} \int_{z_{s}}^{z_{n}}\left[\frac{\partial}{\partial x}\left(\frac{\tilde{P} h^{3}}{12 \mu} \frac{\partial P}{\partial x}\right)+\frac{\partial}{\partial z}\left(\frac{\tilde{P} h^{3}}{12 \mu} \frac{\partial P}{\partial z}\right)\right] d x d z= \\
\int_{x_{w}}^{x_{e}} \int_{z_{S}}^{z_{n}}\left[\frac{U_{x}}{2} \frac{\partial(\tilde{P} h)}{\partial x}+\frac{\partial(P h)}{\partial t}\right] d x d z
\end{gathered}
$$

This leads to the following nonlinear equation:

$$
\begin{aligned}
\tilde{P}_{e} \beta_{e}\left(P_{E}-P_{P}\right)- & \tilde{P}_{w} \beta_{w}\left(P_{P}-P_{W}\right)+\tilde{P}_{n} \beta_{n}\left(P_{N}-P_{P}\right) \\
& -\tilde{P}_{S} \beta_{S}\left(P_{P}-P_{S}\right)=\gamma_{e} \tilde{P}_{e}-\gamma_{w} \tilde{P}_{w}+\frac{\partial P h}{\partial t} \vartheta_{P}
\end{aligned}
$$

where $\vartheta_{P}=\left(x_{e}-x_{w}\right)\left(z_{n}-z_{s}\right)$ and

$$
\beta_{e}=\frac{h_{e}^{3}}{12 \mu} \frac{z_{n}-z_{S}}{x_{E}-x_{p}}, \beta_{w}=\frac{h_{w}{ }^{3}}{12 \mu} \frac{z_{n}-z_{S}}{x_{P}-x_{W}}
$$




$$
\begin{aligned}
& \beta_{n}=\frac{h_{n}{ }^{3}}{12 \mu} \frac{x_{e}-x_{w}}{z_{N}-z_{P}}, \quad \beta_{s}=\frac{h_{s}{ }^{3}}{12 \mu} \frac{x_{e}-x_{w}}{z_{P}-z_{S}} \\
& \gamma_{e}=\frac{U_{x} h_{e}}{2}\left(z_{n}-z_{s}\right), \gamma_{w}=\frac{U_{x} h_{w}}{2}\left(z_{n}-z_{s}\right)
\end{aligned}
$$

The "upwinded" pressures $\widetilde{P}$ write:

$$
\begin{aligned}
& \tilde{P}_{e, n}=P_{P} \delta_{e, n}+P_{E, N}\left(1-\delta_{e, n}\right) \\
& \tilde{P}_{w, s}=P_{W, S} \delta_{w, s}+P_{P}\left(1-\delta_{w, s}\right) \\
& \left\{\begin{array}{l}
\delta_{e, w, s, n}=1 \text { if } u_{e, w, s, n} \geq 0 \\
\delta_{e, w, s, n}=0 \text { otherwise }
\end{array}\right.
\end{aligned}
$$

Equation (4) is written in a more general form:

$a_{E} P_{E}+a_{W} P_{W}+a_{N} P_{N}+a_{S} P_{S}-a_{P} P_{P}=b_{P}+\frac{\partial P h}{\partial t} \vartheta_{P}$

with

$a_{E}=\tilde{P}_{e} \beta_{e}, a_{W}=\tilde{P}_{w} \beta_{w}, a_{N}=\tilde{P}_{n} \beta_{n}, a_{S}=\tilde{P}_{s} \beta_{s}$

$a_{P}=a_{E}+a_{W}+a_{N}+a_{S}$

$b_{P}=\gamma_{e} \widetilde{P}_{e}-\gamma_{w} \tilde{P}_{w}$

The presence of the groove creates a film thickness discontinuity depicted in Figure 7. The film thickness discontinuity must coincide with a cell face. Full conservative discretized equations are obtained by imposing the flow rate conservation on both sides of the cell face carrying the discontinuity ([6], [7]). For example, if the face " $e$ " of the cell carries a discontinuity, then the mass conservation between cells " $P$ " and " $E$ " leads to:

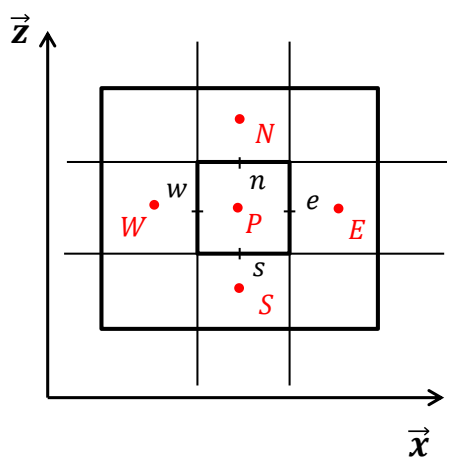

Figure 6 The rectangular control volume

$$
\underbrace{-\frac{h_{e, d}^{3}}{12 \mu}\left(\frac{\partial P}{\partial x}\right)_{e, d}+\frac{U_{x} h_{e, d}}{2}}_{q_{e}^{(P)}}=\underbrace{-\frac{h_{e, g}^{3}}{12 \mu}\left(\frac{\partial P}{\partial x}\right)_{e, g}+\frac{U_{x} h_{e, g}}{2}}_{q_{w}^{(E)}}
$$

where $e, g$ indicates the left side of the face $e$ and $e, d$ its right side. Thus $h_{e, g}$ is the film height on the left side of face $e$ and $h_{e, d}$ is the film height on its right side.

The pressure derivatives are approximated with first order differences:

$$
\left(\frac{\partial P}{\partial x}\right)_{e, d}=\frac{P_{e}-P_{p}}{x_{e}-x_{P}},\left(\frac{\partial P}{\partial x}\right)_{e, g}=\frac{P_{E}-P_{e}}{x_{E}-x_{e}}
$$

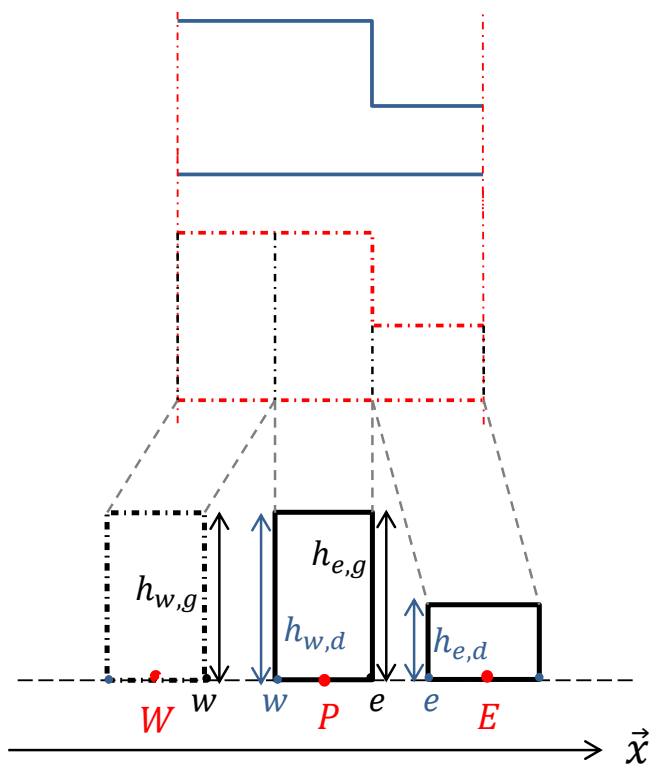

Figure 7 The film thickness discontinuity in $\vec{x}$ direction between successive discretization cells

It is then possible to calculate the pressure on face $e$ from the conservation eq. (14).

$$
\begin{aligned}
& P_{e}=\frac{C_{P}^{e}}{C_{P}^{e}+C_{E}^{w}} P_{P}+\frac{C_{E}^{w}}{C_{P}^{e}+C_{E}^{w}} P_{E}+\frac{U_{x}}{2} \frac{\left(h_{e, d}-h_{e, g}\right)}{C_{P}^{e}+C_{E}^{w}} \\
& C_{P}^{e}=\frac{h_{e, d}^{3}}{12 \mu\left(x_{e}-x_{P}\right)}, C_{E}^{w}=\frac{h_{e, g}^{3}}{12 \mu\left(x_{E}-x_{e}\right)}
\end{aligned}
$$

This pressure value is used for expressing the flow rate at cell face $e$,

$$
\begin{aligned}
& q_{e}^{(P)}=-\tilde{C}_{e}\left(P_{E}-P_{P}\right)+\frac{U_{x}}{2} \tilde{h}_{e} \\
& \tilde{C}_{e}=\frac{C_{P}^{e} C_{E}^{w}}{C_{P}^{e}+C_{E}^{w}}, \tilde{h}_{e}=\frac{C_{P}^{e} h_{e, g}+C_{E}^{w} h_{e, d}}{C_{P}^{e}+C_{E}^{w}}
\end{aligned}
$$

The cell face flow rate given in eq. (18) depends only on the adjacent cell pressures and the film thickness discontinuity is intrinsically taken into account. A similar approach would lead to the pressure at cell face $w$ and the pressures on the cell faces $s$ and $n$ for any discontinuity in the $\vec{z}$ direction. Equation (10) with coefficients taking into account film thickness discontinuities is:

$\tilde{a}_{E} P_{E}+\tilde{a}_{W} P_{W}+\tilde{a}_{N} P_{N}+\tilde{a}_{S} P_{S}-\tilde{a}_{P} P_{P}=\tilde{b}_{P}+\frac{\partial(P h)}{\partial t} \vartheta_{P}$ 
The flow rates and the corresponding coefficients are given in the Annex.

\section{The unstructured triangular grid}

The unstructured grid depicted in Figure 5 is obtained from a Delaunay triangulation generated by using a freeware the code Freefem++ [8]. The discretization cell is depicted in Figure 8. The generalized compressible Reynolds equation for a triangular cell is deduced from the Cartesian one following the Green-Ostrogradski formula.

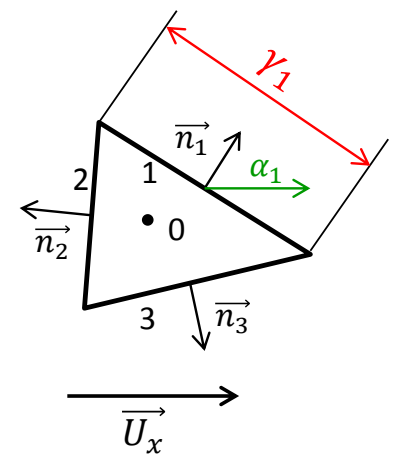

Figure 8 The triangular discretization cell

$$
\int_{V} \ddot{\nabla} \overrightarrow{\dot{m}} d V=\int_{S} \overrightarrow{\dot{m}} \cdot \vec{n} d S
$$

where, $\ddot{\nabla}=\partial / \partial x+\partial / \partial z, \vec{m}=\left(\dot{m}_{x}, \dot{m}_{z}\right)$ and:

$$
\dot{m}_{x}=-\frac{\tilde{P} h^{3}}{12 \mu} \frac{\partial P}{\partial x}+\frac{U_{x}}{2} \frac{\partial(\tilde{P} h)}{\partial x}, \dot{m}_{z}=-\frac{\partial}{\partial z}\left(\frac{\tilde{P} h^{3}}{12 \mu} \frac{\partial P}{\partial z}\right)
$$

Thus the Reynolds equation can be written as

$$
\int_{\Gamma} \frac{\tilde{P} h^{3}}{12 \mu} \frac{\partial P}{\partial n} d \gamma=\int_{\Gamma} \frac{\tilde{P} h}{2} \vec{U}_{x} \cdot \vec{n} d \gamma+\int_{V} \frac{\partial \tilde{P} h}{\partial t}
$$

For a triangular cell, the discretization leads to

$$
\sum_{i=1}^{3} \frac{\tilde{P}_{i} h_{i}{ }^{3}}{12 \mu}\left(\frac{\partial P}{\partial n}\right)_{i} \gamma_{i}=\sum_{i=1}^{3} \frac{\tilde{P}_{i} h_{i}}{2} U_{x} \gamma_{i} \cos \alpha_{i}+\left(\frac{\partial P h}{\partial t}\right)_{0} \vartheta_{0}
$$

The pressure derivative at the cell face is approximated as it follows:

$$
\left(\frac{\partial P}{\partial n}\right)_{i}=\frac{P_{i}-P_{0}}{l_{0 i} \cos \left(\overrightarrow{n_{l}}, \overrightarrow{\xi_{l}}\right)}+\frac{P_{B i}-P_{A i}}{\gamma_{i}} \tan \left(\overrightarrow{n_{l}}, \overrightarrow{\xi_{l}}\right)
$$

and a discretized equation is then obtained:

$$
\sum_{i=1}^{3} a_{i} P_{i}-a_{0} P_{0}=b_{0}
$$

with

$$
\begin{aligned}
a_{i} & =\frac{\tilde{P}_{i} h_{i}{ }^{3} \gamma_{i}}{12 \mu l_{0 i} \cos \left(\overrightarrow{n_{l}}, \overrightarrow{\xi_{l}}\right)}, \quad a_{0}=\sum_{i=1}^{3} a_{i} \\
b_{0} & =\sum_{i=1}^{3} \tilde{P}_{i} \frac{h_{i}}{2} U_{x} \gamma_{i} \cos \alpha_{i}-\sum_{i=1}^{3} \widetilde{P}_{i} \frac{h_{i}{ }^{3}}{12 \mu}\left(P_{B i}-P_{A i}\right) \tan \left(\vec{n}_{l}, \vec{\xi}_{l}\right)
\end{aligned}
$$

As for the rectangular grid, the film height discontinuity requires a special treatment. Figure 9 depicts two adjacent cells indexed 0 and 1 . The common cell face corresponds to the film thickness discontinuity. The flow rate conservation on this face leads to:

$$
\underbrace{-\frac{h_{0}{ }^{3}}{12 \mu}\left(\frac{\partial P}{\partial n}\right)_{01}+\frac{h_{0}}{2} \vec{U}_{x} \cdot \vec{n}_{01}}_{q_{01}^{0}}=\underbrace{-\frac{h_{1}{ }^{3}}{12 \mu}\left(\frac{\partial P}{\partial n}\right)_{10}+\frac{h_{1}}{2} \vec{U}_{x} \cdot \vec{n}_{01}}_{q_{01}^{1}}
$$

The pressure $P_{c}$ at the cell face is then deduced.

$$
\begin{aligned}
& P_{c}=\frac{h_{1}{ }^{3}-h_{0}{ }^{3}}{12 \mu\left(C_{1}+C_{0}\right)} \frac{P_{B}-P_{A}}{l_{A B}} \tan \left(\vec{n}_{01}, \vec{\xi}_{01}\right) \\
& +\frac{h_{0}-h_{1}}{2\left(C_{1}+C_{0}\right)} U_{x} \cos \alpha+\frac{C_{0} P_{0}+C_{1} P_{1}}{C_{1}+C_{0}} \\
& C_{0}=\frac{h_{0}{ }^{3}}{12 \mu l_{0 c} \cos \left(\vec{n}_{01}, \vec{\xi}_{01}\right)} \quad C_{1}=\frac{h_{1}{ }^{3}}{12 \mu l_{1 c} \cos \left(\vec{n}_{01}, \vec{\xi}_{01}\right)}
\end{aligned}
$$

The pressure derivatives on the adjacent face are then expressed in each cell by using $P_{c}$. After rearrangement, the terms of eq. (26) are: ${ }^{1}$

$$
\begin{aligned}
a_{i}= & \frac{\tilde{P}_{i} h_{0 i}{ }^{3} \gamma_{i}}{12 \mu l_{0 i} \cos \left(\overrightarrow{n_{l}}, \overrightarrow{\xi_{l}}\right)} \frac{C_{i i}}{C_{i i}+C_{0 i}} \\
b_{0} & =\sum_{i=1}^{3} \widetilde{P}_{l} \frac{h_{0 i}}{2} U_{x} \gamma_{i} \cos \alpha_{i} \\
& -\sum_{i=1}^{3} \widetilde{P}_{l} \frac{h_{0 i}{ }^{3}}{12 \mu}\left(P_{B i}-P_{A i}\right) \tan \left(\overrightarrow{n_{l}}, \overrightarrow{\xi_{l}}\right) \\
& -\sum_{i=1}^{3} \widetilde{P}_{l} \frac{h_{0 i}{ }^{3}}{12 \mu} \gamma_{i} \frac{h_{0 i}-h_{i i}}{2\left(C_{i i}+C_{0 i}\right)} \frac{U_{x} \cos \alpha_{i}}{l_{0 c_{i}} \cos \left(\vec{n}_{l}, \vec{\xi}_{l}\right)}
\end{aligned}
$$

${ }^{1}$ For a more general expression and to avoid confusion $h_{i j}$ will represent the height of the edge $j$ belonging to the cell $i$. 

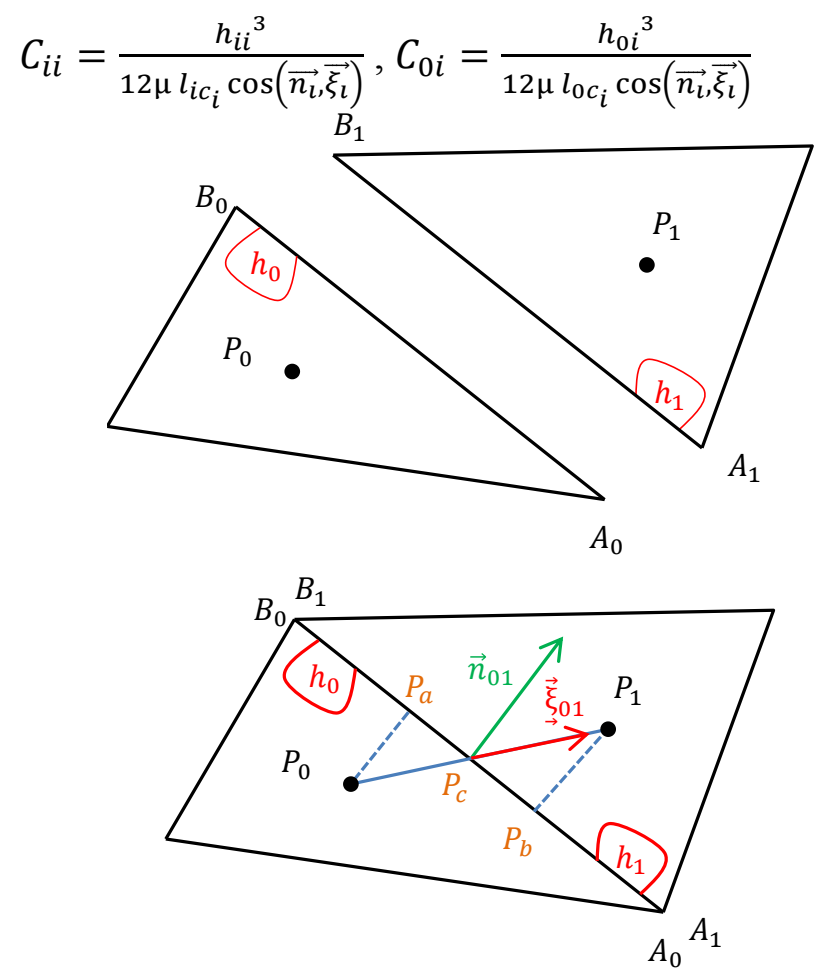

Figure 9 The film thickness discontinuity between two adjacent triangular grid cells

\section{The unsteady effect in the Reynolds equation}

As stated before, for a grooved rotor, the pressure field is unsteady. The implicit backward Euler method was considered for solving the unsteady Reynolds equation and therefore the time dependent term was written as:

$$
\left[\frac{\partial(P h)}{\partial t}\right]_{P}=\frac{P_{P}^{(n)} h_{P}^{(n)}-P_{P}^{(n-1)} h_{P}^{(n-1)}}{\Delta t}
$$

The eq. (20) is then :

$$
\begin{gathered}
\tilde{a}_{E}{ }^{(n)} P_{E}{ }^{(n)}+\tilde{a}_{W}{ }^{(n)} P_{W}{ }^{(n)}+\tilde{a}_{N}{ }^{(n)} P_{N}{ }^{(n)}+\tilde{a}_{S}{ }^{(n)} P_{S}{ }^{(n)} \\
-\tilde{a}_{P}{ }^{\prime} P_{P}{ }^{(n)}=\tilde{b}_{P}{ }^{\prime}
\end{gathered}
$$

With

$$
\begin{aligned}
& \tilde{a}_{P}{ }^{\prime}=\tilde{a}_{P}+\frac{h_{P}{ }^{(n)} \vartheta_{P}}{\Delta t} \\
& \tilde{b}_{P}{ }^{\prime}=\tilde{b}_{P}{ }^{(n)}-\frac{P_{P}{ }^{(n-1)} h_{P}{ }^{(n-1)}}{\Delta t} \vartheta_{P}
\end{aligned}
$$

while all the other terms are unchanged. Equations (36) are non-linear due to the presence of "upwinded" terms $\tilde{P}$. The non-linear system of equations (26) is solved using the NewtonRaphson algorithm at each time step.
The time derivative involves the film heights and pressures at the centers of the cells at the present moment $P_{P}{ }^{(n)}$ and $h_{P}{ }^{(n)}$, but also at the previous instant $P_{P}{ }^{(n-1)}, h_{P}{ }^{(n-1)}$. The groove moves with a constant distance $d$ from one instant to the other.

$$
d=U_{x} \Delta t
$$

This requires a new mesh generation at each time step. Thus, an interpolation of the pressure and of the heights between the current grid and the previous one must be performed. The pressure values are easily interpolated because they represent a $C^{0}$ field (continuous) on the integration domain. The interpolation of the film heights is difficult because of the discontinuity created by the groove. It is then imperative to divide the pad domain into subdomains delimited by the groove (Figure 10) and interpolate each zone separately.

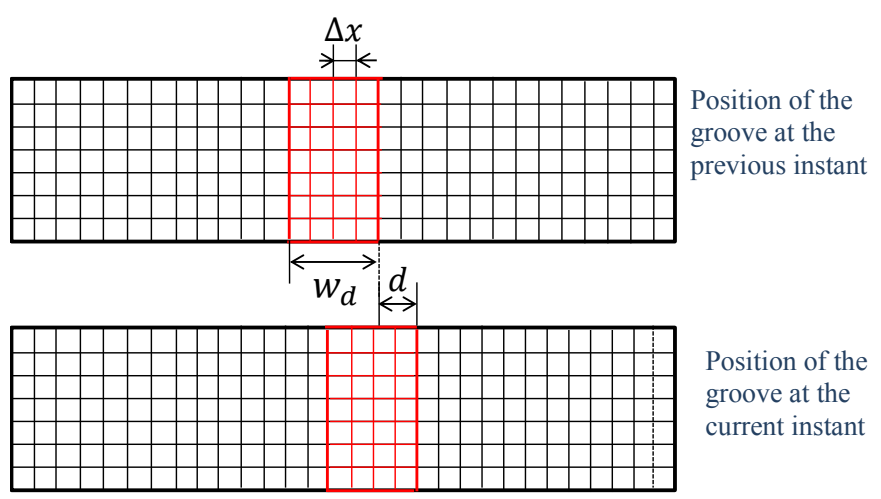

Figure 10 Position of the groove at two successive instants

The reference grid step $\Delta x$ that allows a regular mesh generation at each time step is based on the width of the groove $w_{g}$

$$
\Delta x=\frac{w_{g}}{n}
$$

where $n$ is an integer. A geometrical constraint was then developed for the discretization. It links the displacement of the groove during one step and the grid step. For the rectangular grid, this condition yields:

$$
\Delta x<d=U_{x} \Delta t<w_{g}
$$

and for the triangular grid:

$$
\Delta x<d=U_{x} \Delta t<\frac{w_{g}}{\sin \beta}
$$

The time step is thus intimately linked to the grid step. This condition was needed to avoid situations where only a single grid cell contains the height of the groove. In this case, a discontinuity could induce numerical instabilities. 


\section{RESULTS AND DISCUSSIONS}

The surfaces of the pad and of the runner were considered parallel and $1 \mu \mathrm{m}$ spaced. The depth of the groove is $10 \mu \mathrm{m}$. The ambient pressure is imposed on all sides of the runner. The viscosity value is $\mu=1,8 \cdot 10^{-5} \mathrm{~Pa} \cdot \mathrm{s}$ and an ambient temperature of $T=293 \mathrm{~K}$.

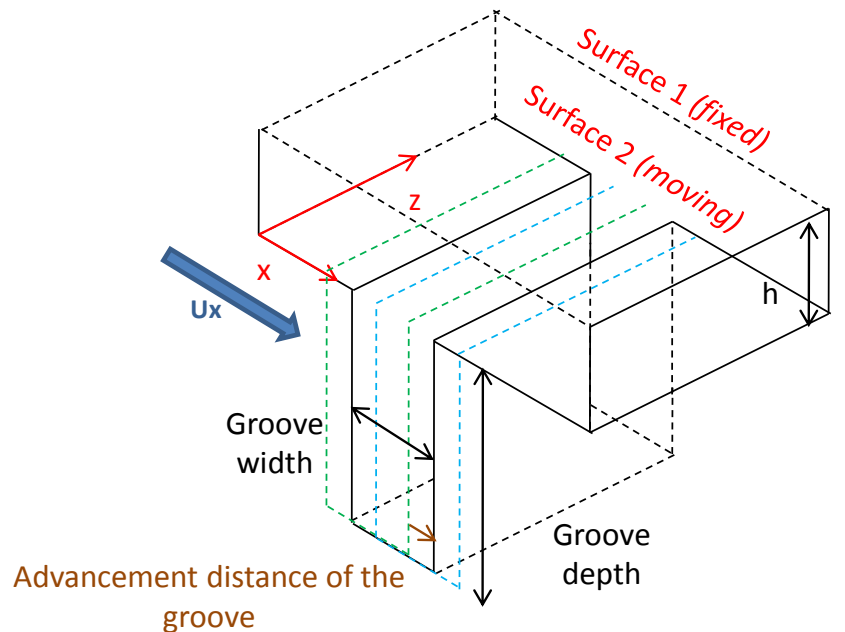

a.

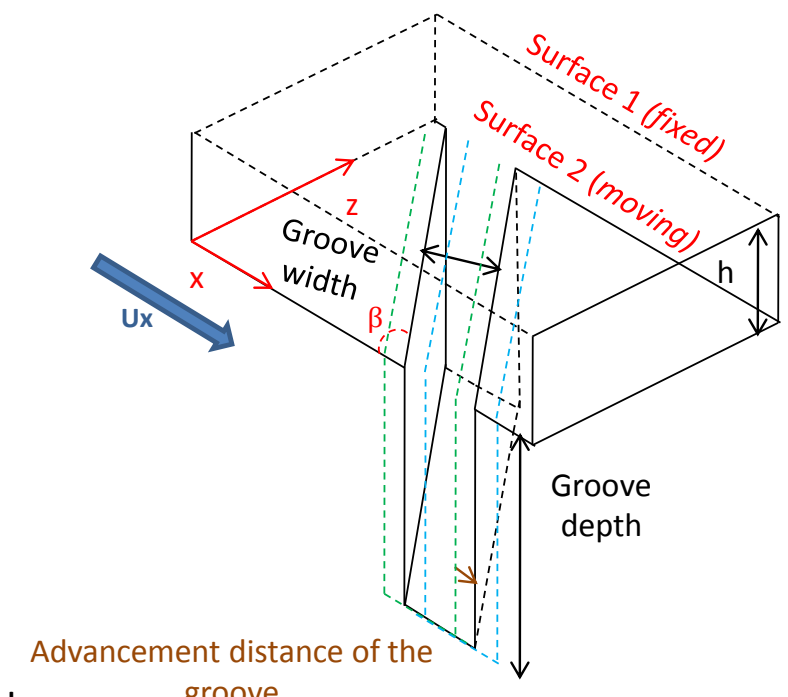

b. Figure 11 The computational domain for: $a-$ the
transversal groove, $b$. - the inclined groove

Figure 11a and Figure 11b show the computational domain for the transversal and for the inclined moving groove. The corresponding grids are shown in Figure 5a and Figure 5b.

The results were obtained considering a moving groove entering and leaving the pad periodically, from left to right (Figure 12). A new groove enters under the pad when the current groove leaves the pad. This means that two successive grooves are spaced by the pad length. The velocity of the runner ranged from $20 \mathrm{~m} / \mathrm{s}$ to $150 \mathrm{~m} / \mathrm{s}$ and the integration time step varied accordingly from $10^{-4} \mathrm{~s}$ to $10^{-5} \mathrm{~s}$.
The main parameter considered to characterize the results is the compressibility number:

$$
\Lambda=\frac{6 \mu U_{x} L_{x}}{P_{a t m} h^{2}}
$$

The results obtained for the transversal and the inclined groove are quantitatively and qualitatively different although

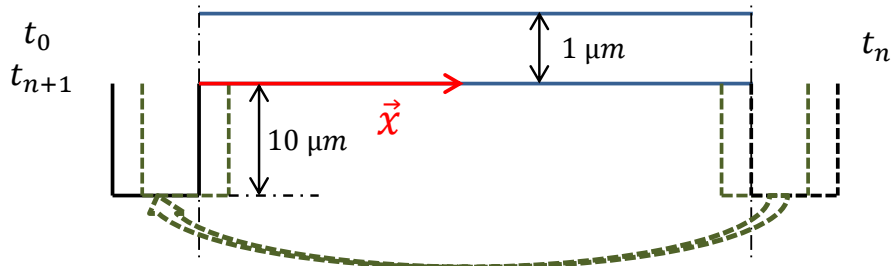

Figure 12 Periodic displacement of the moving groove
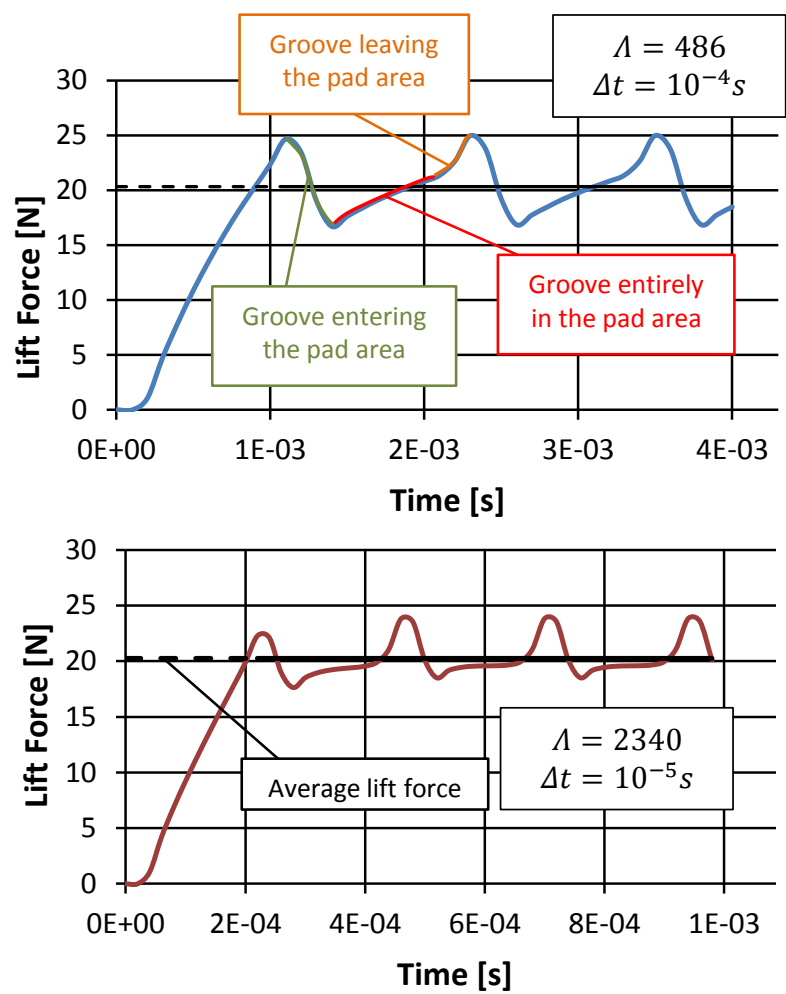

Figure 13 Lift force generated by a transversal groove

the groove had the same length and depth in both cases.

For the transversal groove, the results depicted in Figure 13 show a periodically unsteady lift force established after a short transient lap of time. The lift force varies synchronously with the position of the groove. The period averaged value of the lift force for a compressibility number $\Lambda=486$ is $20 N$. For a compressibility number $\Lambda=2340$ the shape of the pressure variation is more flat but the average value of the lift force is still $20 \mathrm{~N}$. The shear force on the moving surface (Figure 14) is also synchronous with the position of the groove position. Its average values increase following the same ratio as the compressibility number (i.e. $\sim 1 / 5$ ). 
The pressure variation for the inclined groove and a compressibility number $\Lambda=486$ is depicted in Figure 15 . The results show a lift following a double loading period, thus the frequency of the lift force is twice the frequency of the groove. Its period averaged value is $17,5 \mathrm{~N}$.

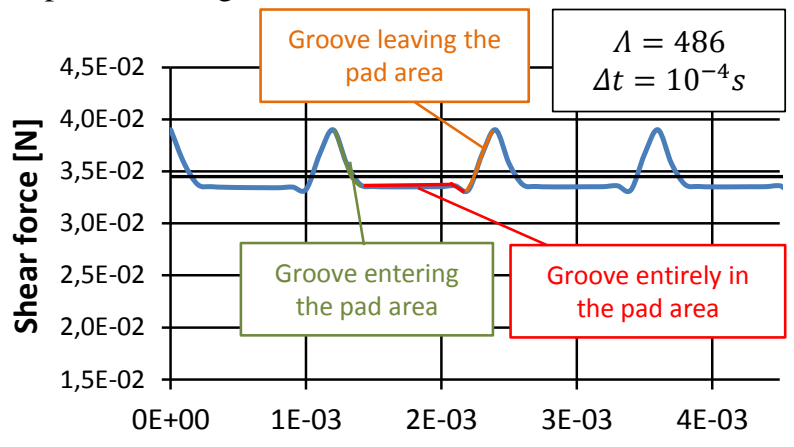

Time $[s]$

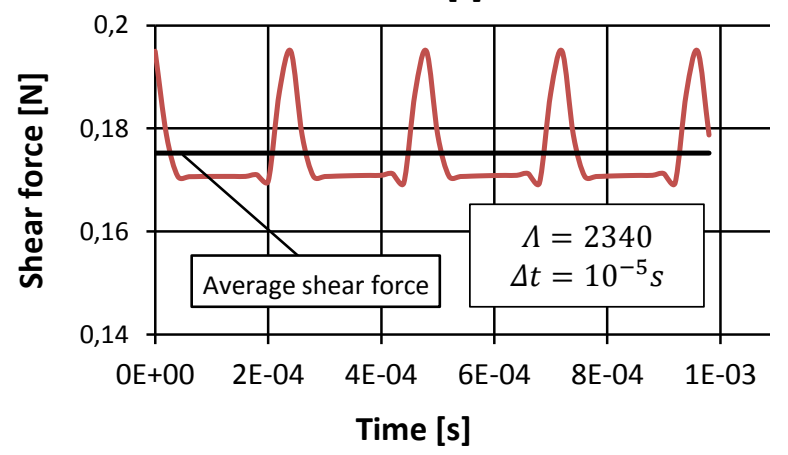

Figure 14 Shear force generated on the moving surface by a transversal groove

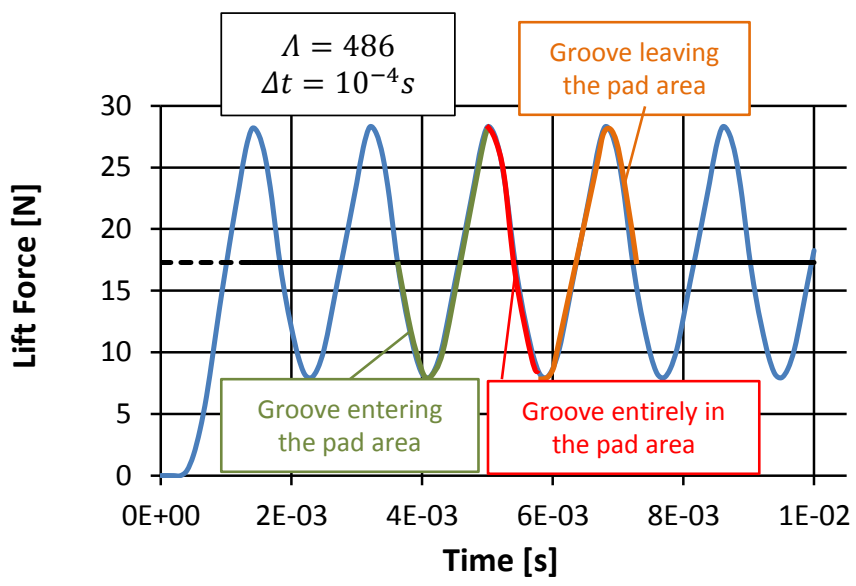

Figure 15 Lift force generated by an inclined groove

The shear force variation on the moving surface depicted in Figure 16 is also twice the frequency of the groove with an average value of $3,210^{-2} \mathrm{~N}$ that is somewhat lower than for a transversal groove $\left(3,510^{-2} \mathrm{~N}\right)$ operating under the same conditions.

The pressure variation for the inclined groove can be decomposed in three phases and many steps. During the first phase, the groove enters under the pad.
First, only one edge of the groove intersects the surface of the pad (Figure 17a). The pressure diminishes during this phase and increases during the second step (Figure 17b) when the second edge of the groove starts intersecting the pad.

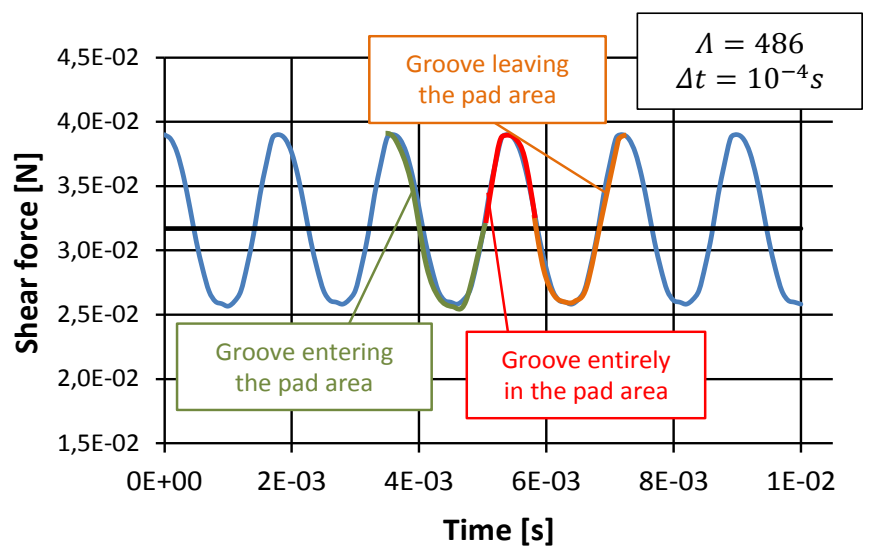

Figure 16 Shear force generated on the moving surface by an inclined groove

During the second phase (Figure 17c), both edges of the groove intersect the long edges of the pad and the lift decreases.

During the third phase, the groove leaves the pad and the variation of the lift is opposite to the entry phase. This phase has also two steps. During the first step (Figure 17d), the pressure increases when one edge of the groove approaches the edge of the pad and then diminishes during the second step (Figure 17e) when the second edge of the groove leaves the pad.

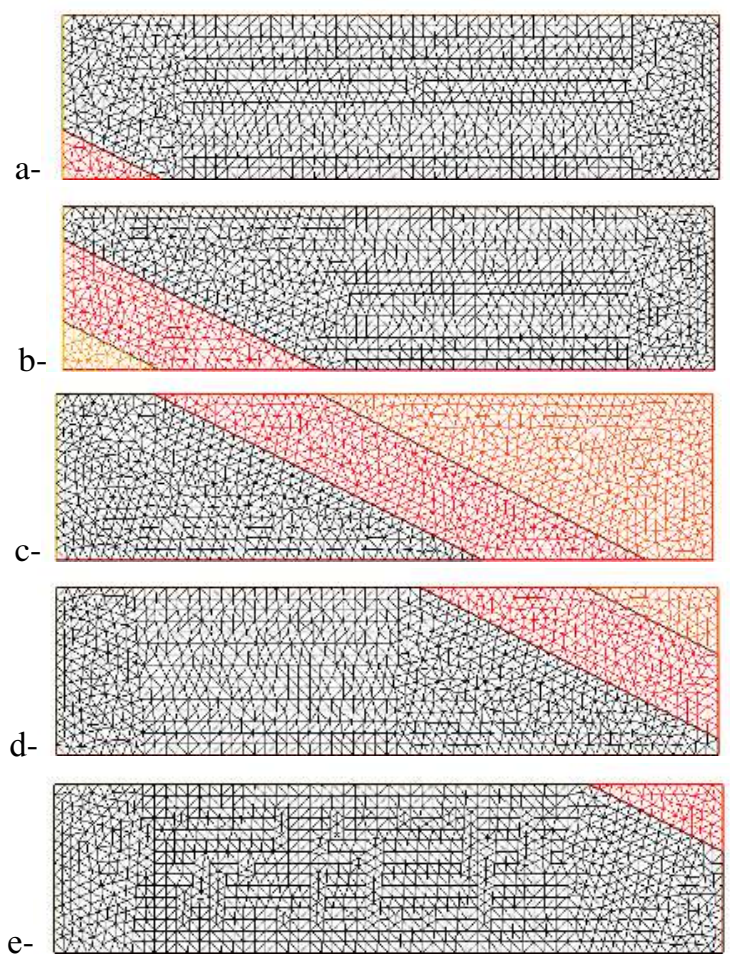


Figure 17 The successive positions of the inclined groove under the pad

Results obtained for the inclined groove and an increased compressibility number of $\Lambda=2340$ are depicted in Figure 18 .

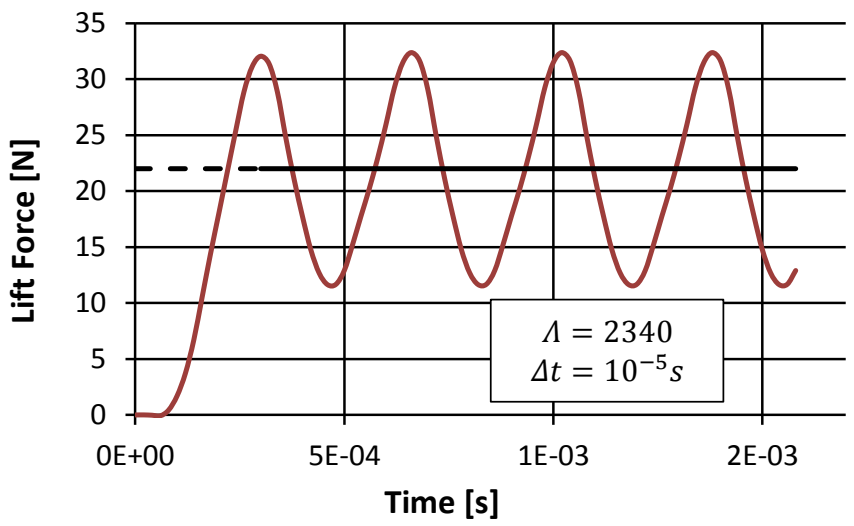

Figure 18 Lift generated by an inclined groove

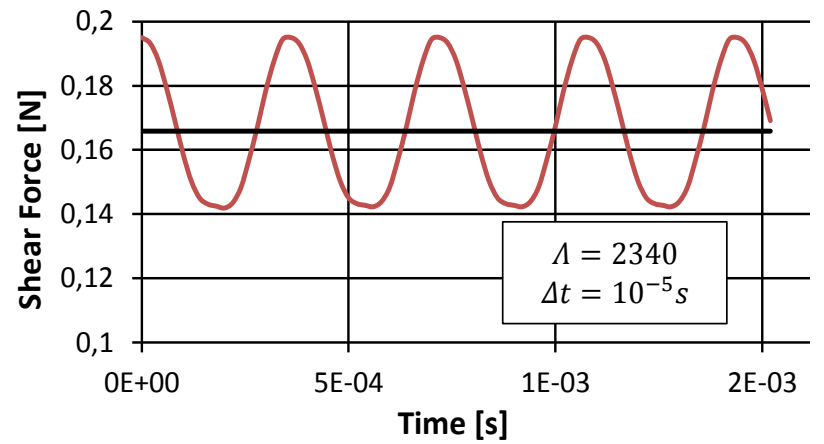

Figure 19 Shear force generated on the moving surface by an inclined groove

The time averaged lift force is $22 \mathrm{~N}$. The average shear force on the moving surface (Figure 19) is again somewhat lower compared to the transversal one.

The variation of the average lift forces with the compressibility numbers for both the inclined and the transversal groove are presented in Figure 20. It is noticed that the lift forces tend to asymptotic values for compressibility number larger than 5000. The inclined groove tends to generate a higher lift force than its kin for the same geometrical characteristics and operating conditions, which is an interesting fact that may play in its favor in practical applications.

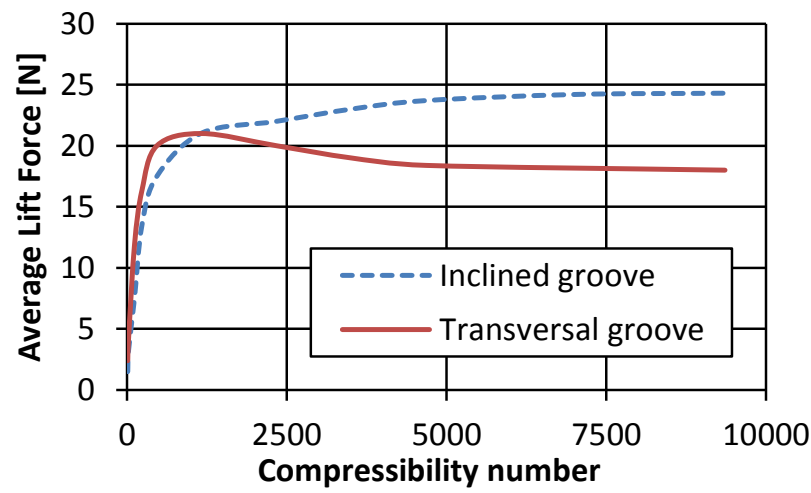

Figure 20 Average lift force as a function of the compressibility number for the inclined and the transversal groove

\section{CONCLUSION}

The analyses of a smooth segmented seal with a grooved runner need to take into account the unsteady Reynolds equation and the thin film discontinuities. Both the transversal and the inclined grooves generate a time averaged lift but the variation of the unsteady force is different. This result is quite unexpected because in both cases (transversal and inclined groove) only a single groove is present under the pad at every moment. The double load period obtained in the case of an inclined groove is an interesting result that should be extended to the case of two or more grooves simultaneously present under the pad. Regarding the average value of the lift, the inclined groove generated a higher force for high compressibility numbers, which might be an interesting feature for practical applications.

\section{ACKNOWLEDGMENTS}

The authors would like to acknowledge SAFRAN Aircraft Engines for financing this work.

\section{REFERENCES}

[1] Burcham R. E., 1978, "Liquid Rocket Engine Turbopump Rotating-Shaft Seals," NASA Lewis Research Center, Cleveland, Ohio, NASA SP-8121.

[2] Burcham R. E., 1983, "High-speed crygoenic self-acting shaft seals for liquid rocket turbopumps," NASA Lewis Research Center, Cleveland, Ohio, NASA CR-168194.

[3] Arghir M., Mariot A., 2017, "Theoretical analysis of the static characteristics of the carbon segmented seal," ASME Journal Tribology, vol. 139.

[4] Bonneau D., Huitric J., Tournerie B., 1993, "Finite element analysis of grooved gas thrust bearings and grooved gas face seals," ASME Journal Tribology, vol. 115.

[5] Lehn A., Shweizer B., 2006, "Generalized Reynolds equation for fluid film problems with arbitrary boundary conditions: application to double-sided spiral groove thrust bearings," Arch Appl Mech, vol. 86. 
[6] Arghir M., Alsayed A., Nicolas D., 2002, "The Finite Volume Solution of Lubrication's Reynolds Equation with Film Discontinuities," International Journal of Mechanical Sciences, vol. 44, pp. 2119, 2132.

[7] Arghir M., Le Lez S., Frene J., 2006, "Finite volume solution of the compressible Reynolds equation- linear and non linear analysis of gas bearings," Proc. IMechE Part J: Journal of Engineering Tribology, vol. 220, pp. 617-627.

[8] Hecht, F., 2012, "New development in FreeFem++", J. Numer. Math. 20, no. 3-4, 251-265. 65Y15 


\section{ANNEX A}

Terms and coefficients of eq. (20)

$$
\begin{aligned}
& \tilde{a}_{E}=\tilde{P}_{e} \tilde{\beta}_{e}, \quad \tilde{a}_{W}=\tilde{P}_{w} \tilde{\beta}_{w} \\
& \tilde{a}_{N}=\tilde{P}_{n} \tilde{\beta}_{n}, \quad \tilde{a}_{S}=\tilde{P}_{s} \tilde{\beta}_{s} \\
& \tilde{a}_{P}=\tilde{a}_{E}+\tilde{a}_{W}+\tilde{a}_{N}+\tilde{a}_{S} \\
& \tilde{b}_{P}=\tilde{\gamma}_{e} \tilde{P}_{e}-\tilde{\gamma}_{w} \tilde{P}_{w}
\end{aligned}
$$

where

$\tilde{\beta}_{e}=\tilde{C}_{e}\left(z_{n}-z_{s}\right), \tilde{\beta}_{w}=\tilde{C}_{w}\left(z_{n}-z_{s}\right)$

$\tilde{\beta}_{n}=\tilde{C}_{n}\left(x_{e}-x_{w}\right), \tilde{\beta}_{s}=\widetilde{C}_{s}\left(x_{e}-x_{w}\right)$

$\tilde{\gamma}_{e}=\frac{U_{x} \widetilde{h}_{e}}{2}\left(z_{n}-z_{s}\right) \quad \tilde{\gamma}_{w}=\frac{U_{x} \widetilde{h}_{w}}{2}\left(z_{n}-z_{s}\right)$

and

$\tilde{C}_{e}=\frac{C_{P}^{e} C_{E}^{w}}{C_{P}^{e}+C_{E}^{w}}, \tilde{h}_{e}=\frac{C_{P}^{e} h_{e, g}+C_{E}^{w} h_{e, d}}{C_{P}^{e}+C_{E}^{w}}$

$\tilde{C}_{w}=\frac{C_{P}^{w} C_{W}^{e}}{C_{P}^{w}+C_{W}^{e}}, \tilde{h}_{w}=\frac{C_{P}^{w} h_{w, d}+C_{w}^{e} h_{w, g}}{C_{P}^{w}+C_{W}^{e}}$

$\tilde{C}_{n}=\frac{C_{P}^{n} C_{N}^{s}}{C_{P}^{n}+C_{N}^{s}}, \quad \tilde{h}_{n}=\frac{C_{P}^{n} h_{n, g}+C_{N}^{S} h_{n, d}}{C_{P}^{n}+C_{N}^{s}}$

$\widetilde{C}_{s}=\frac{C_{P}^{S} C_{S}^{n}}{C_{P}^{w}+C_{S}^{n}}, \tilde{h}_{s}=\frac{C_{P}^{S} h_{s, d}+C_{s}^{n} h_{s, g}}{C_{P}^{S}+C_{S}^{n}}$

with

$C_{P}^{w}=\frac{h_{w, g}^{3}}{12 \mu\left(x_{P}-x_{w}\right)}, C_{W}^{e}=\frac{h_{w, d}^{3}}{12 \mu\left(x_{w}-x_{W}\right)}$

$C_{P}^{S}=\frac{h_{s, g}^{3}}{12 \mu\left(x_{P}-x_{S}\right)}, \quad C_{S}^{n}=\frac{h_{s, d}^{3}}{12 \mu\left(x_{S}-x_{S}\right)}$

And the flow rates are:

$q_{e}^{(P)}=-\tilde{C}_{e}\left(P_{E}-P_{P}\right)+\frac{U_{x}}{2} \tilde{h}_{e}$
$q_{e}^{(P)}=-\tilde{C}_{e}\left(P_{E}-P_{P}\right)+\frac{U_{x}}{2} \tilde{h}_{e}$ 